Tecno Lógicas

ISSN 0123-7799

Vol. 17, No. 32, pp. 47-55

Enero-junio de 2014
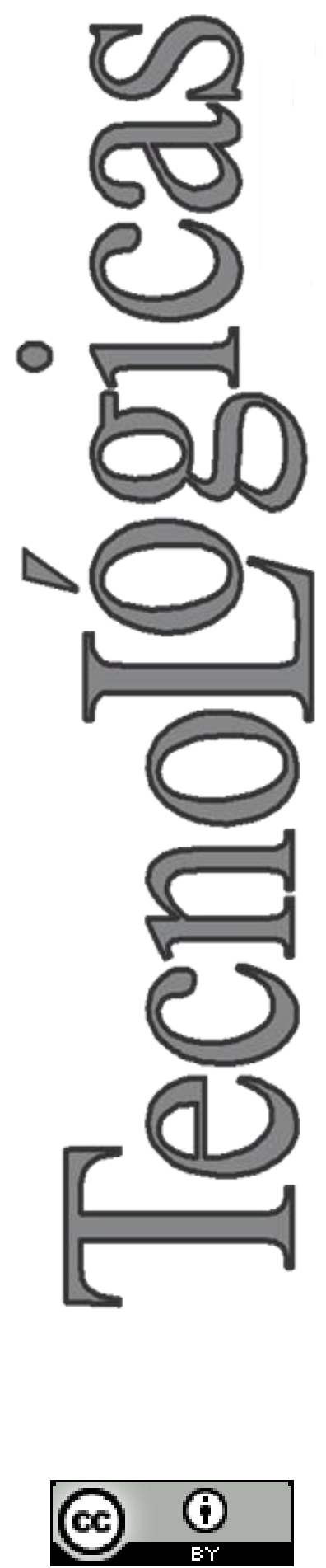

\section{Cálculo del umbral para detección de la onda $R$ del complejo cardiaco}

\section{Threshold calculation for $R$ wave detection in complex cardiac}

\author{
Javier E. González-Barajas ${ }^{1}$
}

Recibido: 10 de octubre de 2013, Aceptado: 20 de diciembre de 2013

Como citar / How to cite

J. E. González-Barajas, "Cálculo del umbral para detección de la onda $\mathrm{R}$ del complejo cardiaco", Tecno Lógicas, vol. 17, no. 32, pp. 47-55, 2014.

1 Magister en Ingeniería, Facultad de Ingeniería Electrónica, División de Ingenierías, Universidad Santo Tomás, Bogotá-Colombia, javiergonzalezb@usantotomas.edu.co 


\section{Resumen}

La señal electrocardiográfica es una señal eléctrica con una amplitud de $1 \mathrm{mV}$ aproximadamente y componentes espectrales entre 0,7 y $100 \mathrm{~Hz}$. El electrocardiograma representa el comportamiento eléctrico del corazón humano y está conformado principalmente por un grupo de ondas denominado el complejo cardiaco. Las ondas que componen el complejo cardiaco son: P, Q, R, S y T. La onda R corresponde a la onda positiva de mayor amplitud de la señal electrocardiográfica y el tiempo entre cada onda permite el cálculo de la frecuencia cardiaca instantánea. Para el cálculo del tiempo entre cada onda $\mathrm{R}$ es necesario la implementación de un sistema de filtrado que permita una atenuación de las componentes espectrales que no pertenecen a esta forma de onda. Posteriormente se procede a un proceso de umbralización que consiste en generar una señal binaria que toma el valor de uno en la muestra que registra la existencia de una onda $\mathrm{R}$ y cero en las demás muestras. El objetivo de este trabajo es presentar los resultados obtenidos al implementar un algoritmo para el establecer del umbral basado en el cálculo del histograma de la señal electrocardiográfica que ha sido previamente tratada a través de un sistema basado en bancos de filtros.

\section{Palabras clave}

Onda R, complejo cardiaco, banco de filtros, histograma, umbral.

\section{Abstract}

The electrocardiographic signal is an electrical signal and its amplitude is $1 \mathrm{mV}$ approximately and spectral components between 0.7 and $100 \mathrm{~Hz}$. The electrocardiographic signal represents the electrical behavior of the human heart and it has a group of waves called the cardiac complex. Waves comprising the cardiac complex are: $\mathrm{P}, \mathrm{Q}, \mathrm{R}, \mathrm{S}$ and $\mathrm{T}$. The R-wave corresponds to the positive wave of greater amplitude of the electrocardiographic signal and the time between each wave allows the calculation of instantaneous heart rate. The calculation of the time between $R$ wave requires implementation of a filtering system that allows an attenuation of the spectral components that do not belong to this waveform. Then proceed to a thresholding process that consists of generating a binary signal which takes the value of one in the sample that records the wave $\mathrm{R}$ and zero in the other samples. The principal goal of this paper is to present the results to implement an algorithm for setting the threshold based on the calculation of the histogram of the electrocardiographic signal that has been previously addressed through a system based on filter banks.

\section{Keywords}

$\mathrm{R}$ wave, complex cardiac, filter bank, histogram, threshold. 


\section{INTRODUCCIÓN}

El registro electrocardiográfico (ECG) es una señal con una amplitud de aproximadamente $1 \mathrm{mV}$ pico a pico y sus componentes espectrales están contenidas entre 0,7 y $100 \mathrm{~Hz}$ [1]. El ECG está compuesto por un grupo de ondas que constituyen el complejo cardiaco. Estas ondas se denominan acorde a su orden de ocurrencia: P, Q, R, S y T [2]. La Fig. 1 contiene un ejemplo de un ECG y las diferentes ondas que lo componen.



Fig. 1. Registro ECG y las respectivas ondas que componen esta señal. Fuente: Autor

Como se puede observar en la Fig. 1, la onda $\mathrm{R}$ corresponde a la onda positiva de mayor amplitud, está precedida por la onda $\mathrm{Q}$ y antecede a la onda S. La onda R posee una gran importancia en el análisis del ECG ya que representa una referencia para la estimación de la frecuencia cardiaca instantánea [3].

Para la detección de la onda $\mathrm{R}$ se han propuesto diferentes estrategias para resaltar las componentes espectrales que se encuentran en un ancho de banda entre los 10 y $20 \mathrm{~Hz}$ [4]. También se han obtenido éxitos a través del uso de redes neuronales y transformada wavelet [5], implementación de primeras derivadas [6], transformada curvelet [7], interpolación basada en curvas splines [8] y descomposición empírica [9]. Los procesos de detección de la onda $\mathrm{R}$ proporcionan como resultado el realce de esta componente del complejo cardiaco en el dominio del tiempo. El siguiente paso consiste en la umbralización de la onda $\mathrm{R}$ y tiene el objetivo de encontrar la ubicación en el tiempo de cada una de estas ondas. Se han propuesto métodos para la umbralización de la onda $\mathrm{R}$ basados en valores que varían acorde a los cambios de amplitud de la señal [10].

El objetivo principal de este trabajo es la implementación de una técnica para mejorar el proceso de umbralización de la onda $\mathrm{R}$ basada en el cálculo del histograma del registro ECG [11], [12]. Con la finalidad de obtener una mejora en el proceso se incluye previamente el realce de la onda $R$, a través de la estrategia denominada Banco de Filtros en el Dominio del Tiempo.

Con respecto a metodologías presentadas en la literatura se han desarrollado metodologías para el proceso de umbralización que exigen la implementación de procesos computacionales de mayor complejidad. En el caso del uso de redes neuronales, se han registrado éxitos en la implementación de sistemas basados en micro controladores [13]. También se han diseñado métodos que exigen la implementación de filtros digitales de alto orden para el desplazamiento de la línea de base y evitar la alteración del complejo QRS [14], aplicaciones de la transformada wavelet [8] y transformada curvelet.

El método propuesto en este trabajo exige en primera instancia la implementación de un banco de filtros basado en ecuaciones de diferencias y la generación de un histograma que implica operaciones de comparación y cuantificación de ocurrencias.

\section{METODOLOGÍA}

Los experimentos llevados a cabo en este trabajo fueron realizados usando la base de datos de señales fisiológica Physionet [15]. Para lo cual se tomaron señales ECG de pacientes con ritmo sinusal normal. En primera instancia se ha desarrollado una metodología para la detección de la onda $R$. Posteriormente se describe el proceso de 
umbralización de esta componente del complejo cardiaco a través del cálculo del histograma. Con respecto a las herramientas computacionales, se han realizado diferentes pruebas utilizando computadores personales con diferentes tipos de procesadores Intel Pentium, Intel Core Duo e Intel i7. La herramienta informática utilizada para el diseño y pruebas de los algoritmos fue el asistente matemático Matlab®.

\subsection{Detección de la onda $\mathbf{R}$}

Con la finalidad de poder resaltar la onda R del ECG y facilitar su detección se ha utilizado la estrategia de bancos de filtros en el dominio del tiempo ilustrado en la Fig. 2. El sistema se compone de cuatro filtros pasa banda con anchos de banda consecutivos y tiene el objetivo de determinar el rango de frecuencias donde están localizadas las componentes espectrales asociadas a la onda $\mathrm{R}$ del complejo cardiaco.

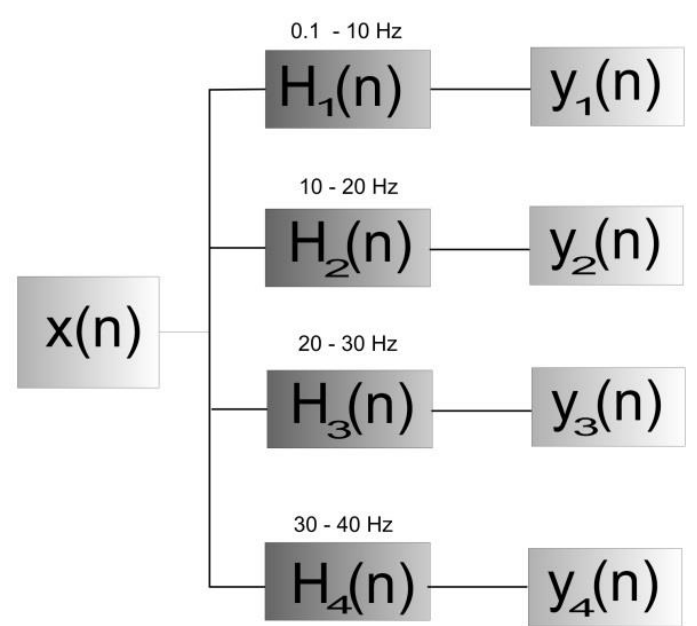

Fig. 2. Banco de filtros en el dominio del tiempo. Fuente: Autor

Cada filtro pasa: $H_{1}(n), H_{2}(n), H_{3}(n)$ y $H_{4}(n)$, está denominado consecutivamente, según el rango de frecuencias. Respectivamente, el resultado que permite obtener el banco de filtros está compuesto de un grupo de señales de salida: $\mathrm{y}_{1}(\mathrm{n}), y_{2}(n), y_{3}(n) \mathrm{y}$ $y_{4}(n)$. Estas señales contienen, en el domi- nio del tiempo, las componentes del registro ECG que están asociadas a cada rango de frecuencias. La señal de salida que presente una onda $\mathrm{R}$ de mayor amplitud, corresponderá al filtro que posee el rango de frecuencias asociadas a esta componente del complejo cardiaco.

\subsection{Cálculo del histograma}

El cálculo del histograma es un proceso que permite cuantificar la frecuencia de ocurrencia de un valor de amplitud del registro ECG. Por lo cual es importante contar con valores de amplitud en formato de número entero. Si el registro ECG está almacenado en un arreglo de datos $y_{n}(n)$, se puede utilizar el algoritmo descrito en la Fig. 3 para obtener una señal con un número entero de amplitudes con un valor mínimo de 0 y un valor máximo igual a 100.

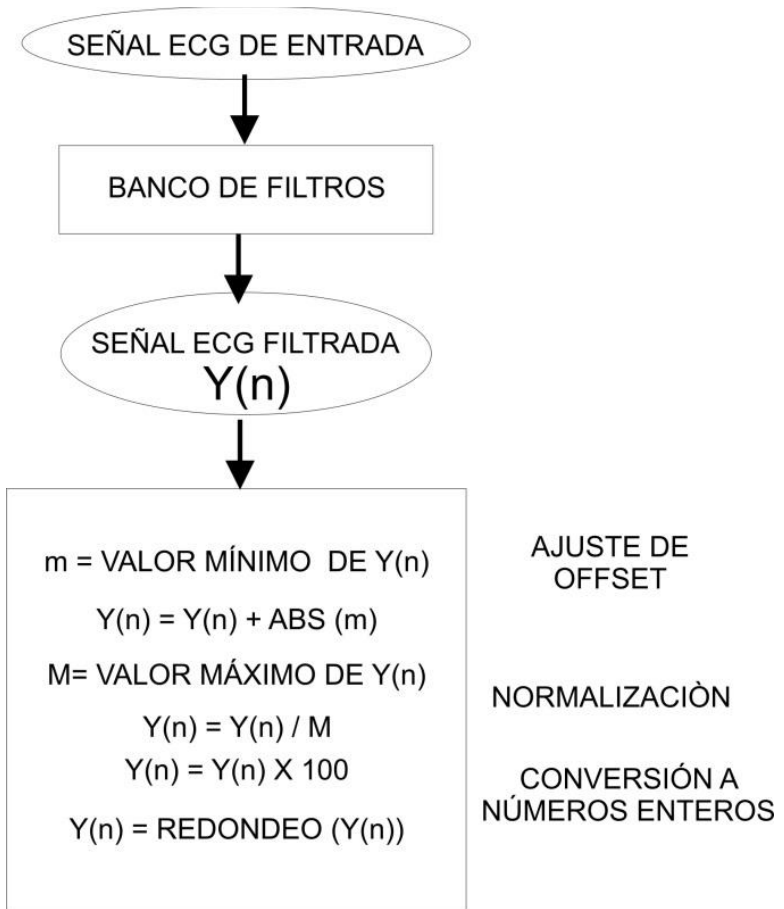

Fig. 3. Ajuste de valores del registro ECG. Fuente: Autor

El algoritmo descrito en la Fig. 3 permite establecer todos los posibles valores de amplitud del registro ECG. Este algoritmo proporciona los parámetros para la cons- 
trucción de la matriz $A$ de dimensiones $F \mathrm{x}$ $C$. Donde $F$ corresponde a los valores posibles de amplitud (0 -100) y $C$ a la cantidad de muestras.

La matriz $A$ contiene solo valores de $0 \mathrm{y}$ 1 , donde el valor de 1 corresponde a la presencia de un valor de amplitud $F$ en la muestra de posición $C$ (ver Fig. 4). La matriz A se construye a partir del siguiente seudocódigo:

$$
\begin{aligned}
& \text { for } i=1: C \\
& \quad q=y(i) ; \\
& A(q, i)=1 \\
& \text { end }
\end{aligned}
$$

Como último paso para la construcción del histograma, se debe proceder a tomar cada fila de la matriz generada y calcular la cantidad de posiciones con valor igual a 1. Este procedimiento da como resultado un arreglo de datos con la frecuencia de ocurrencia de cada valor de amplitud (histograma). La Fig. 4 permite observar un ejemplo del histograma que se obtiene.

\section{RESULTADOS Y DISCUSIÓN}

La Fig. 5 contiene un ejemplo de una señal electrocardiográfica de un paciente con ritmo sinusal normal, con frecuencia de muestreo $f_{s}=333,333 \mathrm{~Hz}$ y adquirida de la base de datos de Physionet [13].

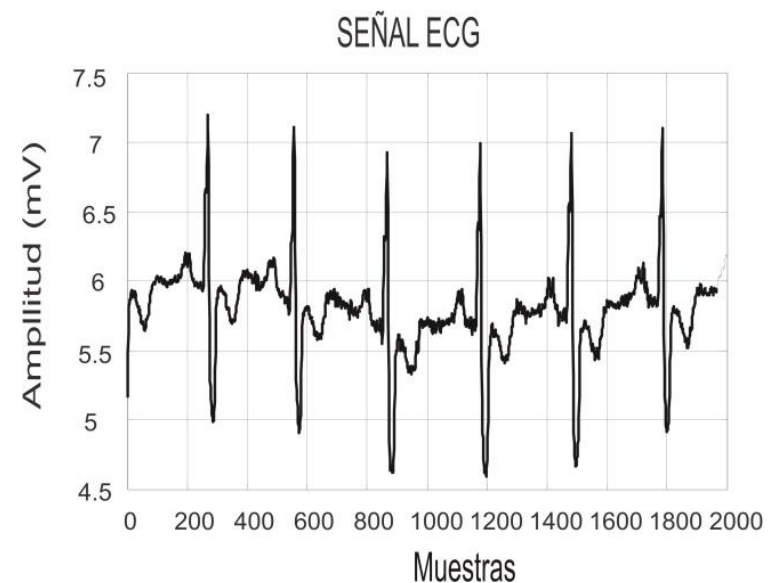

Fig. 5. Señal ECG de muestra $\operatorname{con} f_{s}=333,333 \mathrm{~Hz}$. Fuente: Autor

Con la finalidad de poder encontrar el rango de frecuencias asociadas con la onda $\mathrm{R}$ del complejo cardiaco, se procede al análisis de esta señal bajo el esquema del banco de filtros. El resultado se puede observar en la Fig. 6. El resultado (Fig. 6) indica que en el rango entre 10 y $20 \mathrm{~Hz}$ se puede apreciar un realce de la onda $\mathrm{R}$ del complejo cardiaco. Este experimento indica el rango de frecuencias que contienen la información que caracteriza la onda $R$.

La técnica del banco de filtro ha permitido obtener un criterio base para diseñar un filtro pasa banda con la función de aislar las componentes espectrales que no aportan información para la detección de la onda R. La Fig. 7 contiene el resultado de aplicar a la señal ECG un filtro pasa banda con frecuencias de corte entre 10 y $20 \mathrm{~Hz}$.

\begin{tabular}{|c|c|c|c|c|c|c|c|c|c|c|c|c|c|c|c|}
\hline 0 & 0 & 0 & 0 & 0 & 0 & 0 & 0 & 0 & 0 & 0 & 0 & 0 & 0 & 0 & 0 \\
\hline 0 & 0 & 0 & 0 & 0 & 0 & 1 & 0 & 0 & 0 & 0 & 0 & 0 & 0 & 0 & 0 \\
\hline 0 & 0 & 0 & 0 & 0 & 0 & 0 & 0 & 0 & 0 & 0 & 0 & 0 & 1 & 0 & 0 \\
\hline 0 & 0 & 0 & 0 & 0 & 0 & 0 & 1 & 0 & 0 & 0 & 0 & 0 & 0 & 1 & 0 \\
\hline 0 & 0 & 0 & 0 & 0 & 1 & 0 & 0 & 1 & 0 & 0 & 0 & 1 & 0 & 0 & 1 \\
\hline 0 & 0 & 0 & 0 & 0 & 0 & 0 & 0 & 0 & 0 & 0 & 0 & 0 & 0 & 0 & 0 \\
\hline 0 & 0 & 0 & 0 & 1 & 0 & 0 & 0 & 0 & 1 & 0 & 1 & 0 & 0 & 0 & 0 \\
\hline 0 & 1 & 0 & 1 & 0 & 0 & 0 & 0 & 0 & 0 & 1 & 0 & 0 & 0 & 0 & 0 \\
0 & 0 & 1 & 0 & 0 & 0 & 0 & 0 & 0 & 0 & 0 & 0 & 0 & 0 & 0 & 0 \\
\hline
\end{tabular}

Columnas. $\mathrm{C}=$ Número de muestras que posee la señal ECG.

Fig. 4. Matriz para representación binaria de un registro ECG. Fuente: Autor 

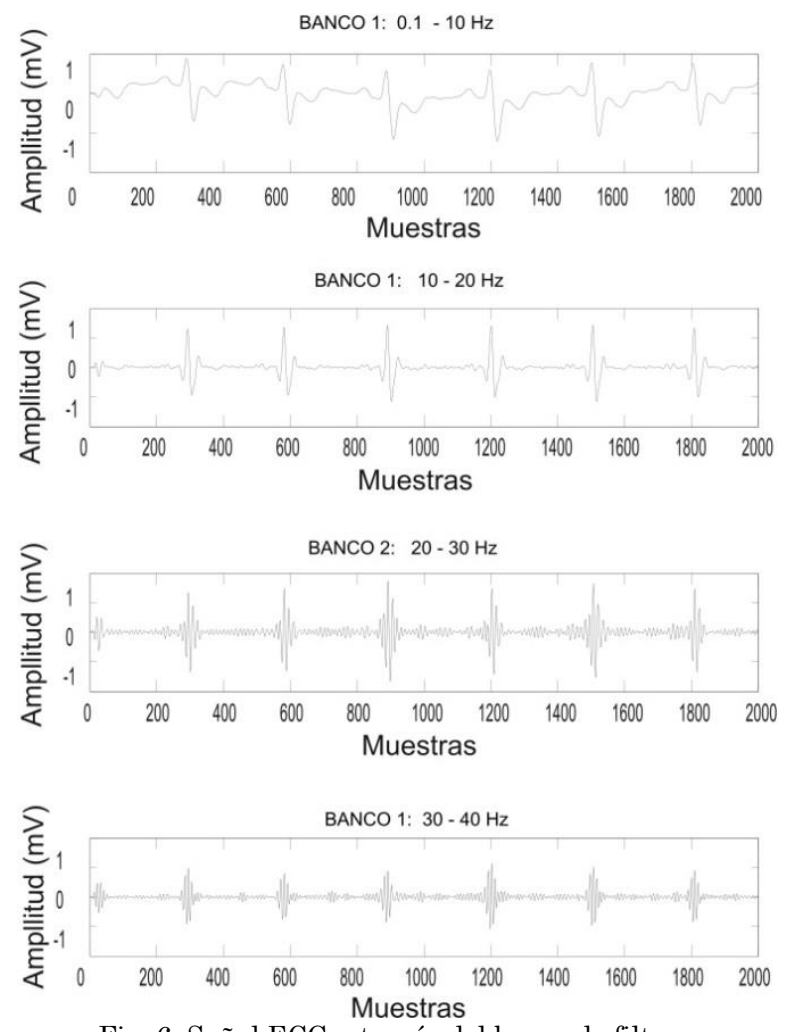

Fig. 6. Señal ECG a través del banco de filtros. Fuente: Autor

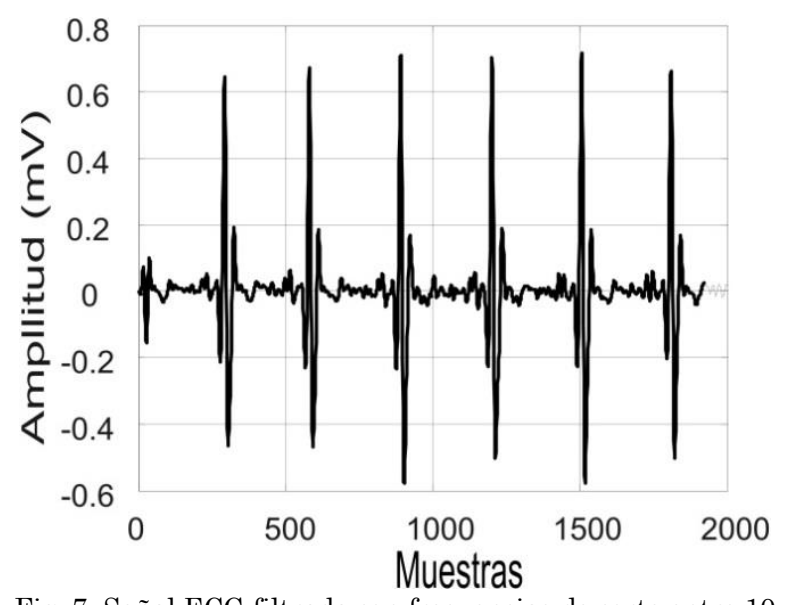

Fig. 7. Señal ECG filtrada con frecuencias de corte entre 10 y $20 \mathrm{~Hz}$. Fuente: Autor

La señal ECG filtrada debe ser sometida al algoritmo de ajuste de valores ilustrado en la Fig. 3. El algoritmo de ajuste permite obtener una señal con los valores adecuados para ser ingresado al proceso de cálculo del histograma. La Fig. 8 permite observar el histograma calculado a la señal ECG ajustada a valores enteros entre $0 \mathrm{y}$ 100.

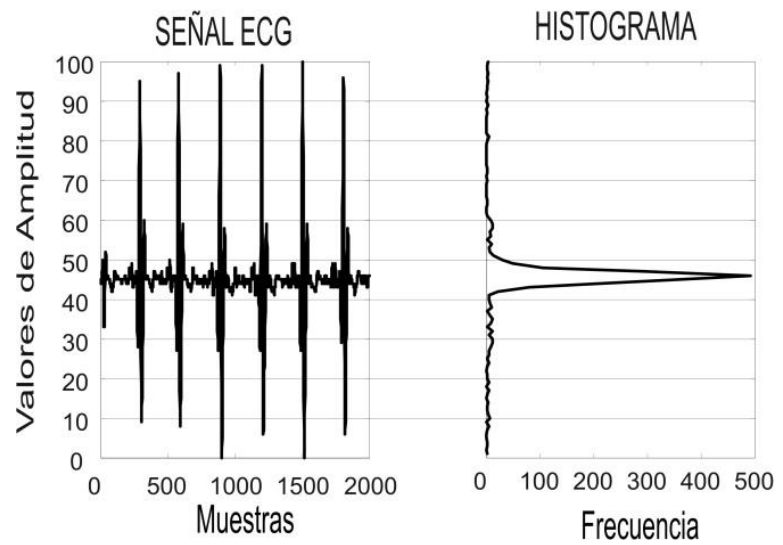

Fig. 8. Señal ECG e Histograma. Fuente: Autor

El histograma obtenido permite obtener una tendencia de la cantidad de apariciones de cada valor de amplitud en cada una de las muestras de la señal ECG. Como se pudo observar en la Fig. 8, el valor de amplitud igual a 45 corresponde al de mayor frecuencia y representa el valor de continua de la señal ECG. Los valores de amplitud entre 50 y 60 presentan el segundo grupo con frecuencia de ocurrencia, correspondiendo a los valores de las ondas $\mathrm{P}$ y $\mathrm{S}$ del complejo cardiaco. Con la información aportada por el histograma se puede encontrar el grupo de valores de amplitud asociados a las ondas $\mathrm{R}$ del registro ECG. Al calcular la segunda derivada del histograma se encuentra una nueva curva ilustrada en la Fig. 9.

El comportamiento de la segunda derivada contiene un valor pico negativo que corresponde al valor de frecuencia de mayor ocurrencia. En la Fig. 9 se puede observar que después de este valor mínimo se puede encontrar un valor de magnitud cero. Este valor igual a cero, comparado con la curva del histograma, corresponde al límite entre los valores asociados a la onda $\mathrm{R}$ y a las ondas $\mathrm{P}$ y $\mathrm{S}$. La ubicación de este valor igual a cero corresponde al umbral del registro ECG bajo estudio.

Con la finalidad de comprobar la utilidad del valor escogido, se procede a realizar el proceso de umbralización de la señal ECG bajo estudio. El resultado se puede verificar en la Fig. 10. 
J. E. González-Barajas / Cálculo del umbral para detección de la onda R del complejo cardiaco

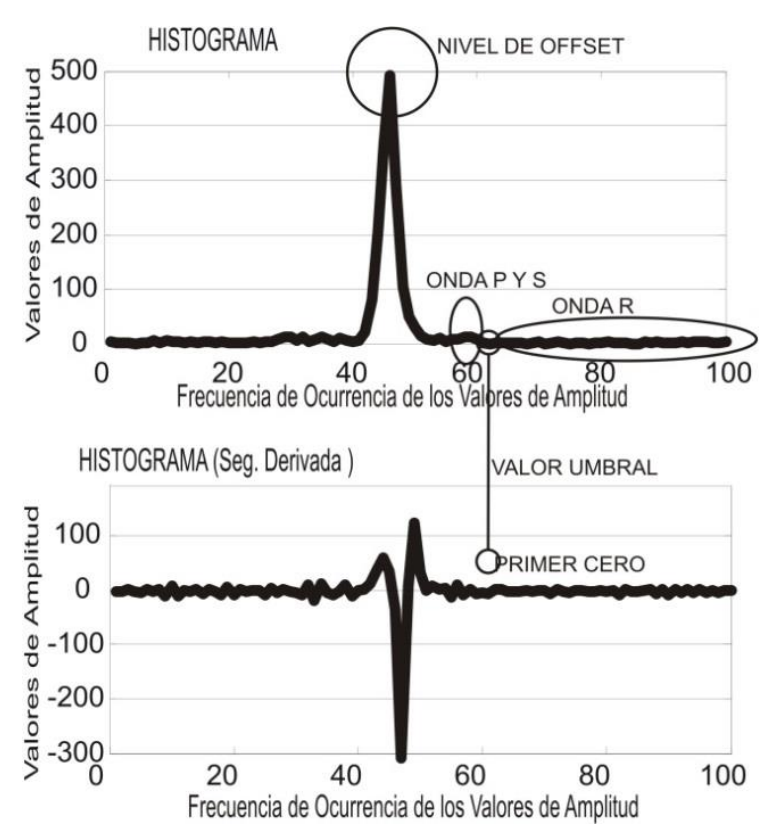

Fig. 9. Histograma y segunda derivada. Fuente: Autor
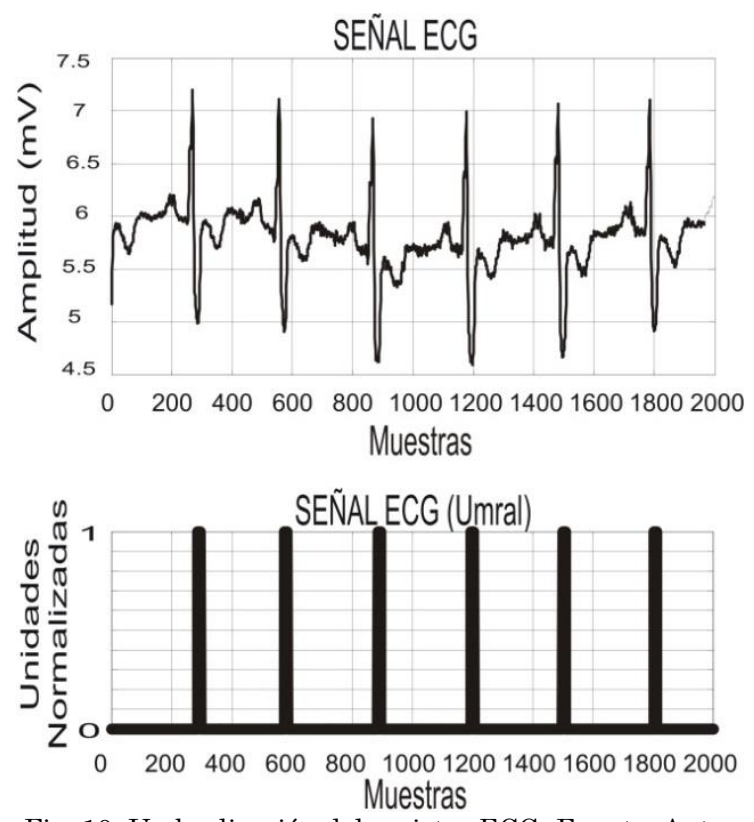

Fig. 10. Umbralización del registro ECG. Fuente: Autor

El resultado contenido en la Fig. 10 demuestra la eficacia del método al detectar correctamente las cinco ondas $\mathrm{R}$ de los complejos cardiacos contenidos en la señal ECG bajo estudio. Las pruebas de esta estrategia se realizaron tomando 5 registros electrocardiográficos con duración de 10 segundos cada uno. Los resultados obtenidos se pueden verificar en la Tabla 1.
Tabla 1. Porcentaje de detección de onda R. Fuente: Autor

\begin{tabular}{ccc}
$\begin{array}{c}\text { Registro ECG } \\
\text { Physionet }\end{array}$ & $\begin{array}{c}\text { Ondas R } \\
\text { encontradas }\end{array}$ & $\begin{array}{c}\text { Ondas R } \\
\text { detectadas }\end{array}$ \\
\hline Reg Num 16265 & 16 & 16 \\
Reg Num 16273 & 16 & 16 \\
Reg Num 16539 & 13 & 13 \\
Reg Num 16795 & 11 & 11 \\
\hline
\end{tabular}

La señales ECG pueden verse alteradas por diferentes tipos de ruidos, por lo cual es necesario contar con ensayos del algoritmo presentado en condiciones en las cuales la señal de prueba puede estar distorsionada. La Fig. 11 contiene una versión de la señal ECG contaminada con ruido de línea de potencia eléctrica y desplazamiento de la línea de base.

\section{SEÑAL ECG con Ruido Simulado}

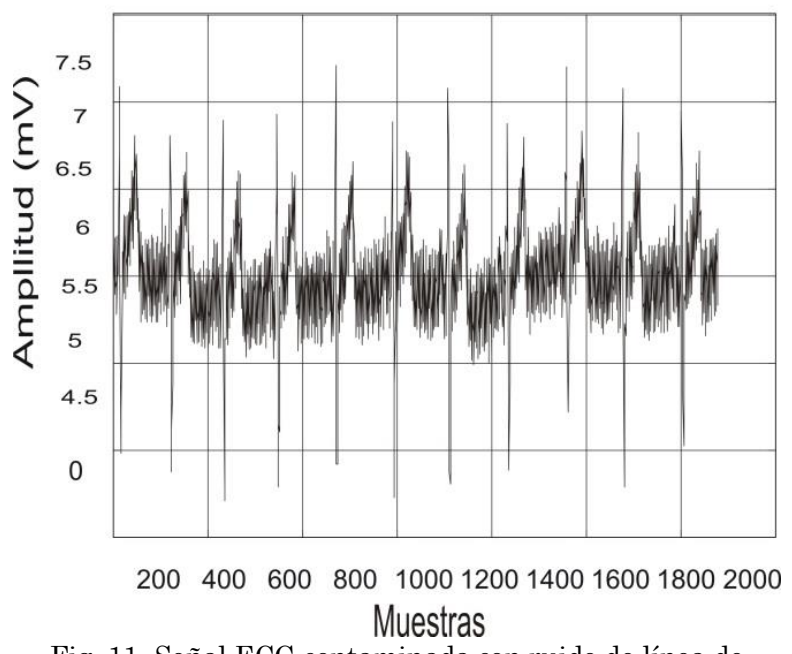

Fig. 11. Señal ECG contaminada con ruido de línea de potencia eléctrica. Fuente: Autor

La Fig. 12 contiene el resultado al realizar el proceso del banco de filtros y posterior umbralización. El resultado permite ver que el banco de filtros se encarga de atenuar las componentes que no pertenecen a la onda $\mathrm{R}$, por lo cual se verifica que en condiciones de ruido se puede obtener una correcta detección de esta onda. La Fig. 12 en su sección inferior contiene el resultado del proceso de umbralización obtenido a partir de la señal filtrada. 

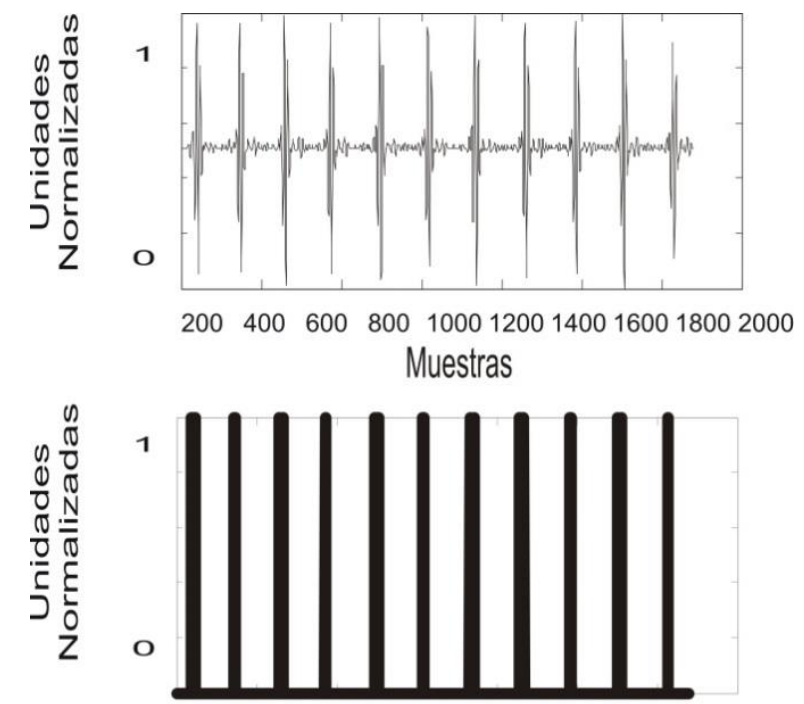

200400600800100012001400160018002000 Muestras

Fig. 12. Proceso de banco de filtro y umbralización en condiciones de ruido. Fuente: Autor

Para medir el tiempo de cómputo de los dos procesos: banco de filtros y umbralización, se ha diseñado un experimento en el cual se tomó una señal de prueba de 200 muestras. Los valores de tiempos obtenidos se ilustran en la Tabla 2 para tres tipos diferentes de procesadores: Intel Pentium, Intel Core Duo e Intel i7.

\begin{tabular}{lcc}
\multicolumn{3}{c}{ Tabla 2. Tiempo de cómputo y eficiencia. Fuente: Autor } \\
\hline $\begin{array}{l}\text { Equipo de } \\
\text { computo }\end{array}$ & $\begin{array}{c}\text { Tiempo ejecución } \\
\text { Banco de filtros }\end{array}$ & $\begin{array}{c}\text { Tiempo ejecución } \\
\text { Umbralización }\end{array}$ \\
\hline $\begin{array}{l}\text { PC basado en } \\
\text { Intel Pentium }\end{array}$ & $0,9 \mathrm{~s}$ & $1,6 \mathrm{~s}$ \\
$\begin{array}{l}\text { PC basado en } \\
\text { Intel Core Duo }\end{array}$ & $0,47 \mathrm{~s}$ & $0,6 \mathrm{~s}$ \\
$\begin{array}{l}\text { PC basado en } \\
\text { Intel i7 }\end{array}$ & $0,3 \mathrm{~s}$ & $0,4 \mathrm{~s}$
\end{tabular}

\section{CONCLUSIONES}

El artículo contiene los resultados obtenidos en la mejora del proceso de umbralizacion de la onda $\mathrm{R}$ del complejo cardiaco a través de la implementación de dos técnicas: Banco de Filtros en el Dominio del Tiempo y Cálculo del Histograma del registro ECG. El banco de filtros ofrece un valor agregado gracias a la capacidad de estimar el rango de frecuencias donde están ubica- das las componentes espectrales asociadas a la onda $\mathrm{R}$ del complejo cardiaco y atenuar las distorsiones causadas por interferencias. Como se pudo ver en los resultados obtenidos, el rango de frecuencia está entre los 10 y $20 \mathrm{~Hz}$. En el caso de que un registro ECG presente un ruido causado por desplazamiento de línea de base y/o provocado por las líneas de potencia eléctrica, las componentes espectrales de estos ruidos están por fuera del rango de frecuencias asociadas a la onda $\mathrm{R}$ del complejo cardiaco.

Los resultados obtenidos al aplicar el cálculo del histograma permitieron obtener de manera automatizada un valor umbral para la onda $\mathrm{R}$ del complejo cardiaco. El valor umbral no depende de un valor empírico que tenga que ser reajustado, ya que el método permite estimarlo a través de la frecuencia de ocurrencia de los valores de amplitud que corresponden a las ondas $\mathrm{R}$ que están en el registro ECG bajo estudio.

Se pudo evidenciar que el algoritmo puede funcionar en diferentes tipos de procesadores y que para los de última generación se pueden obtener bajos tiempos de cómputo.

\section{AGRADECIMIENTOS}

Los resultados presentados son logrados dentro del proyecto de investigación titulado: "Plataforma informática para el análisis de la variabilidad de la frecuencia cardiaca orientado a la prestación de servicios de análisis y cálculo de descriptores", patrocinado por la Unidad de Investigación de la Universidad Santo Tomás (FODEIN 2012).

\section{REFERENCIAS}

[1] J. D. Enderle, S. M. Blanchard, and J. D. Bronzino, Introduction to biomedical engineering. San Diego: Academic Press, 2000, p. 1062.

[2] B. M. Koeppen and B. A. Stanton, Berne y Levy. Fisiología, 6th ed. Elsevier Inc., 2009, p. 848. 
[3] J. G. Barrero, O. L. Rueda, M. Pinto, and J. Gonzáles, "Cardiotacómetro digital: Prototipo para estudio de la variabilidad de la frecuencia cardíaca," Rev. UIS Ing., vol. 2, no. 2, pp. 81-86, Oct. 2011.

[4] W. J. Tompkins, Biomedical Digital Signal Processing. Prentice-Hall, 1993.

[5] S. M. Szilagyi and L. Szilagyi, "Wavelet transform and neural-network-based adaptive filtering for QRS detection," in Proceedings of the 22nd Annual International Conference of the IEEE Engineering in Medicine and Biology Society (Cat. No.00CH37143), 2000, vol. 2, no. 2, pp. 1267-1270.

[6] N. M. Arzeno, Z.-D. Deng, and C.-S. Poon, "Analysis of first-derivative based QRS detection algorithms.," IEEE Trans. Biomed. Eng., vol. 55, no. 2 Pt 1, pp. 47884, Feb. 2008.

[7] J. Lewandowski, H. E. Arochena, R. N. G. Naguib, and K.-M. Chao, "A simple real-time QRS detection algorithm utilizing curve-length concept with combined adaptive threshold for electrocardiogram signal classification," in TENCON 2012 IEEE Region 10 Conference, 2012 , vol. 1 , no. 1, pp. 1-6.

[8] C.-I. Ieong, P.-I. Mak, C.-P. Lam, C. Dong, M.-I. Vai, P.-U. Mak, S.-H. Pun, F. Wan, and R. P. Martins, "A 0.83- $\mathrm{WW}$ QRS detection processor using quadratic spline wavelet transform for wireless ECG acquisition in 0.35- $\mu \mathrm{m}$ CMOS.," IEEE Trans. Biomed. Circuits Syst., vol. 6, no. 6, pp. 586-95, Dec. 2012.

[9] H. Xing and M. Huang, "A New QRS Detection Algorithm Based on Empirical Mode Decomposition," in 2008 2nd International Conference on Bioinformatics and Biomedical Engineering, 2008, vol. 1, no. 1, pp. 693-696.

[10] M. Elgendi, S. Mahalingam, M. Jonkman, and F. De Boer, "A Robust QRS Complex Detection Algorithm Using Dynamic Thresholds," in International Symposium on Computer Science and its Applications, 2008, vol. 1, no. 1, pp. 153-158.

[11] W. Li, X. Chen, and S. Fan, "A histogram and difference based approach for $\mathrm{R}$ wave real time detection," in 2009 IEEE International Conference on Granular Computing, 2009, vol. 1, no. 1, pp. 362-367.

[12] H.-J. Park, D.-U. Jeong, and K.-S. Park, "Automated detection and elimination of periodic ECG artifacts in EEG using the energy interval histogram method.," IEEE Trans. Biomed. Eng., vol. 49, no. 12 Pt 2, pp. 1526-33, Dec. 2002.

[13] P. Kumar, M. Jain, and S. Chandra, "Low Cost, Low Power QRS Detection Module Using PIC," in 2011 International Conference on Communication Systems and Network Technologies, 2011, vol. 1, pp. 414-418.

[14] E. Zeraatkar, S. Kermani, A. Mehridehnavi, and A. Aminzadeh, "Improving QRS detection for artifacts reduction," in 2010 17th Iranian Conference of Biomedical Engineering (ICBME), 2010, vol. 1, no. 1, pp. 1-4.

[15] PhysioNet, "Physiological signal data base." [Online]. Available: www.physionet.org. [Accessed: 15-Dec2013]. 